QMW-00-02

RUNHETC-2000-11

hep-th/0004086

April, 2000

\title{
BPS Supermultiplets in Five Dimensions
}

\author{
C.M. HulL \\ Physics Department, Queen Mary and Westfield College, \\ Mile End Road, London E1 4NS, U.K. \\ and \\ Department of Physics and Astronomy, Rutgers \\ University, Piscataway, New Jersey 08855-0849, USA
}

\begin{abstract}
BPS representations of 5-dimensional supersymmetry algebras are classified. For BPS states preserving 1/2 the supersymmetry, there are two distinct classes of multiplets for $N=4$ supersymmetry and three classes for $N=8$ supersymmetry. For $N=4$ matter theories, the two $1 / 2$ supersymmetric BPS multiplets are the massive vector multiplet and the massive self-dual 2-form multiplet. Some applications to super-Yang-Mills, supergravity and little string theories are considered.
\end{abstract}


Representations of supersymmetry carrying central charges have been extensively studied; see e.g. [1]. The purpose of this note is to clarify some of the issues that arise in $4+1$ dimensions, improving on some misleading statements in the literature, and to classify the possible BPS multiplets. Some standard results are recovered, but some unexpected features are found, such as the existence of three distinct possible representations for massive BPS states in $N=8$ theories preserving $1 / 2$ the supersymmetry.

The $N=2 n$ extended superalgebra in $4+1$ dimensions with scalar central charges has automorphism group $S p(n)=U S p(2 n)$ and the anti-commutator

$$
\left\{Q_{\alpha}^{a}, Q_{\beta}^{b}\right\}=\Omega^{a b}\left(\Gamma^{M} C\right)_{\alpha \beta} P_{M}+C_{\alpha \beta}\left(Z^{a b}+\Omega^{a b} K\right)
$$

Here $\alpha=1, \ldots, 4$ are spinor indices and $a=1, \ldots N$ are $S p(n)$ indices, $C_{\alpha \beta}$ is the charge conjugation matrix and $\Omega^{a b}$ is the symplectic invariant of $S p(n)$. The supercharges $Q_{\alpha}^{a}$ are symplectic Majorana spinors satisfying

$$
(\bar{Q})_{a}^{\alpha}=C^{\alpha \beta} \Omega_{a b} Q_{\beta}^{b}
$$

The central charges $Z^{a b}$ satisfy $Z^{a b}=-Z^{b a}$ and $\Omega_{a b} Z^{a b}=0$. This can be generalised by adding 1 -form and 2-form central charges which are carried by 1,2,3 and 4-branes [2]. In the following, it will be useful to define

$$
W^{a b}=Z^{a b}+\Omega^{a b} K
$$

In super-Yang-Mills theories and in $N=8$ supergravity, the charges $Z^{a b}$ are electric charges while $K$ is carried by solitons in $4+1$ dimensions that arise from lifting instantons in 4 Euclidean dimensions. In $N=4$ supergravity coupled to matter, $K$ is a linear combination of such an instantonic charge and an ordinary electric charge. The massless representations have vanishing central charges $W^{a b}=0$ and are supermultiplets with $2^{N}$ states fitting into representations of the 
little group $S O(3) \times S p(n)$; a list of supergravity and supermatter representations with $N \leq 8$ is given in [1]. For example, the $N=4$ super-Yang-Mills multiplet decomposes into the following representations of $S O(3) \times S p(2)$ :

$$
N=4 S Y M: \quad 2^{4}=(3,1)+(1,5)+(2,4)
$$

and there is a variant $N=4$ super-Yang-Mills multiplet, the $S p(2)$ super-YangMills multiplet, with the following structure:

$$
(3,10)+(1,5)+(1,10)+(1,35)+(2,4)+(2,16)+(2,20)
$$

in which the vector fields are in a $\mathbf{1 0}$ of the R-symmetry group $S p(2) \sim \operatorname{Spin}(5)$. The $N=8$ supergravity multiplet decomposes into the $S O(3) \times S p(4)$ representations:

$$
N=8 \text { SUGRA }: \quad 2^{8}=(5,1)+(3,27)+(1,42)+(4,8)+(2,48)
$$

The general (non-BPS) massive representations have dimension $4^{N}$, but there are shorter representations in which the central charge $W^{a b} \neq 0$ and the mass saturates a BPS bound. The little group for the massive representations is $\operatorname{Spin}(4) \times$ $S p(n)$. The supercharges, which transform as a $(4, n)$ of $\operatorname{Spin}(4,1) \times S p(n)$ and satisfy the reality constraint $(2)$, transform as a $(2,1 ; n)+(1,2 ; n)$ under the little group $S U(2) \times S U(2) \times S p(n)$, giving the 'chiral' supercharges $Q_{ \pm}^{a}$ with $Q_{ \pm}^{a}= \pm \Gamma^{0} Q_{ \pm}^{a}$. In the rest frame with $P^{M}=(M, 0, \ldots, 0)$ (with signature $(+----)$ ), the algebra (1) takes the form

$$
\begin{aligned}
& \left\{Q_{+}, Q_{+}^{\dagger}\right\}=M \mathbb{1}_{2 n \times 2 n}-\hat{W} \\
& \left\{Q_{-}, Q_{-}^{\dagger}\right\}=M \mathbb{1}_{2 n \times 2 n}+\hat{W} \\
& \left\{Q_{+}, Q_{-}^{\dagger}\right\}=0
\end{aligned}
$$

where $\hat{W}=-\Omega^{-1} W$. 
It will be useful to choose a basis in which $W$ is skew-diagonal with skew eigenvalues $\lambda_{1}, \ldots, \lambda_{n}$, so that

$$
W=\Lambda \otimes\left(\begin{array}{cc}
0 & 1 \\
-1 & 0
\end{array}\right)
$$

where $\Lambda$ is the diagonal $n \times n$ matrix whose entries are the eigenvalues

$$
\Lambda=\operatorname{diag}\left(\lambda_{1}, \ldots \lambda_{n}\right)
$$

and the symplectic invariant is

$$
\Omega=\mathbb{I}_{n \times n} \otimes\left(\begin{array}{cc}
0 & 1 \\
-1 & 0
\end{array}\right)
$$

so that $\hat{W}$ is diagonal

$$
\hat{W}=\Lambda \otimes\left(\begin{array}{ll}
1 & 0 \\
0 & 1
\end{array}\right)
$$

with eigenvalues $\lambda_{i}$, each of degeneracy two. Then the BPS bound derived from (7) is

$$
M \geq \lambda, \quad \lambda \equiv \max \left|\lambda_{i}\right|
$$

Note that

$$
K=2 \sum_{i} \lambda_{i}=\operatorname{tr} \hat{W}
$$

so that $\Lambda$ is traceless unless $K \neq 0$. If $Z \neq 0$, then the central charge $Z$ will break the $S p(n)$ symmetry to a subgroup.

Let $r_{+}$be the number of the eigenvalues $\lambda_{i}$ satisfying $\lambda_{i}=\lambda$ and $r_{-}$be the number of $\lambda_{i}$ satisfying $\lambda_{i}=-\lambda$ (so that $r_{+}+r_{-} \leq n$ ). A BPS state satisfying $M=\lambda$ will be invariant under the action of $2 r_{+}$of the 2-component supercharges $Q_{+}$and $2 r_{-}$of the 2 -component supercharges $Q_{-}$, i.e. it is invariant under $4 r_{+}$ 
positive chirality supersymmetries and $4 r_{-}$negative chirality ones. The fraction of supersymmetry preserved is $\nu=\left(r_{+}+r_{-}\right) / 2 n$. Note that $\nu \leq 1 / 2$. The state will then fit into a representation of the supersymmetry algebra generated by the remaining $4 p$ positive chirality supersymmetries and $4 q$ negative chirality ones, with $p=n-r_{+}, q=n-r_{-}$; this will be referred to as a $(p, q)$ massive $D=5$ supermultiplet.

One motivation for this nomenclature is so that the dimensional reduction on a circle of a massless representation of $(p, q)$ supersymmetry in $5+1$ dimensions gives a Kaluza-Klein tower of massive multiplets in $4+1$ dimensions, each of which is a $(p, q)$ massive $D=5$ supermultiplet. (Recall that the $(p, q)$ superalgebra in 6 dimensions has $p$ right-handed symplectic Majorana-Weyl supercharges and $q$ left-handed ones.) The momentum in the circle direction gives the central charge carried by the massive multiplet on dimensional reduction. The $(p, q)$ multiplet decomposes into representations of the 'little group' $\operatorname{Spin}(4) \times S p(p) \times S p(q)$.

There are similar multiplets in other odd dimensions; for example, in 9 dimensions, there are $(1,0),(2,0)$ and $(1,1)$ massive BPS supersymmetry multiplets, such as those arising from the Kaluza-Klein modes of circle compactifications of $(1,0)$, $(2,0)$ and $(1,1)$ supersymmetric theories in $D=10$, respectively. The BPS states of type II string theory compactified to 9 dimensions were considered in [3], where three types of multiplets were identified, the KKA, the KKB and the intermediate multiplets; these are $(1,1),(2,0)$ and $(2,1)$ multiplets, respectively.

The massive multiplets with $N \leq 8$ and $p \geq q$ are listed in table 1 . There are two types of $1 / 2$ supersymmetric BPS multiplets with $N=4$, the $(1,1)$ and $(2,0)$ multiplets, and three types with $N=8$, the $(4,0),(3,1)$ and $(2,2)$ multiplets. 


\begin{tabular}{|c|c|c|c|c|}
\hline$N$ & $r_{+}$ & $r_{-}$ & $(p, q)$ & $\nu$ \\
\hline $2 n$ & 0 & 0 & $(n, n)$ & 0 \\
\hline 2 & 0 & 1 & $(1,0)$ & $1 / 2$ \\
\hline 4 & 0 & 2 & $(2,0)$ & $1 / 2$ \\
\hline 4 & 1 & 1 & $(1,1)$ & $1 / 2$ \\
\hline 4 & 0 & 1 & $(2,1)$ & $1 / 4$ \\
\hline 6 & 0 & 1 & $(3,2)$ & $1 / 6$ \\
\hline 6 & 0 & 2 & $(3,1)$ & $1 / 3$ \\
\hline 6 & 1 & 1 & $(2,2)$ & $1 / 3$ \\
\hline 6 & 0 & 3 & $(3,0)$ & $1 / 2$ \\
\hline 6 & 1 & 2 & $(2,1)$ & $1 / 2$ \\
\hline 8 & 0 & 1 & $(4,3)$ & $1 / 8$ \\
\hline 8 & 0 & 2 & $(4,2)$ & $1 / 4$ \\
\hline 8 & 1 & 1 & $(3,3)$ & $1 / 4$ \\
\hline 8 & 0 & 3 & $(4,1)$ & $3 / 8$ \\
\hline 8 & 1 & 2 & $(3,2)$ & $3 / 8$ \\
\hline 8 & 0 & 4 & $(4,0)$ & $1 / 2$ \\
\hline 8 & 1 & 3 & $(3,1)$ & $1 / 2$ \\
\hline 8 & 2 & 2 & $(2,2)$ & $1 / 2$ \\
\hline
\end{tabular}

Table 1 BPS multiplets in 5 dimensions. The $(p, q)$ BPS multiplets for $N \leq 8$ supersymmetry in 5 dimensions with $p \geq q$ are listed. They preserve a fraction $\nu$ of the $4 N$ supersymmetries and fit into massive multiplets of the active $(p, q)$ supersymmetry.

Consider first $N=4$ supersymmetry, with two eigenvalues $\lambda_{1}, \lambda_{2}$. If $\lambda_{1} \neq \lambda_{2}$, then the central charge $Z \neq 0$ and breaks the $\operatorname{Sp}(2) \sim \operatorname{Spin}(5) \mathrm{R}$-symmetry to $\operatorname{Sp}(1) \times \operatorname{Sp}(1) \sim \operatorname{Spin}(4)$. If for $i=1,2, M>\left|\lambda_{i}\right|$, no supersymmetry is preserved and the multiplet is the general $(2,2)$ massive one. If $M=\left|\lambda_{1}\right|>\left|\lambda_{2}\right|$, then $1 / 4$ supersymmetry is preserved (i.e. 4 of the 16 supersymmetries) and the multiplet is $(2,1)$ or $(1,2)$ depending on the sign of $\lambda_{1}$. For $1 / 2$ supersymmetric states, $M=\left|\lambda_{1}\right|=\left|\lambda_{2}\right|$. The $(1,1)$ BPS multiplets have $\Lambda=\operatorname{diag}(\lambda,-\lambda)$ and so $K=0$ but the central charge $Z^{a b}$ is non-zero, breaking the $S O(5)$ R-symmetry to $S O(4)$, while the $(2,0)$ multiplets have $\Lambda=-\operatorname{diag}(\lambda, \lambda)$ and so $Z^{a b}=0$ and $K=-\lambda$ is non-zero, preserving the full $S O(5)$. The $(1,1)$ supersymmetric massive vector 
multiplet has the $S U(2) \times S U(2) \times S p(1) \times S p(1)$ content

$$
(2,2 ; 1,1)+(1,1 ; 2,2)+(2,1 ; 1,2)+(1,2 ; 2,1)
$$

while there is a $(2,0)$ massive multiplet with the $S U(2) \times S U(2) \times S p(2)$ content

$$
(3,1 ; 1)+(1,1 ; 5)+(2,1 ; 4)
$$

The massive covariant field whose physical degrees of freedom are in the $(3,1 ; 1)$ representation is a self-dual massive 2 -form field, i.e. a 2 -form gauge field $B$ satisfying the self-duality constraint

$$
d B=m * B
$$

where $*$ is the Hodge dual and $m$ is the mass. Such massive self-dual $n$-form fields in $2 n+1$ dimensions occur in various supergravity theories and their compactifications; for example, a massive self-dual 4-form occurs in the (2,0) massive multiplet in 9 dimensions and arises as a Kaluza-Klein mode for the self-dual 4-form of $D=10$ IIB supergravity when compactified on a circle. Note that these massive vector and massive 2 -form multiplets in $D=5$ both have the same massless limit, which is the massless vector multiplet (4) (after dualising the massless 2-form to a 1-form gauge field), so that in $D=5$ there are two distinct massive generalisations of the Yang-Mills multiplet. There are also $(1,1)$ and $(2,0)$ massive supergravity and gravitino multiplets that arise as Kaluza-Klein modes in the compactification of 6-dimensional supergravity theories on a circle.

The $N=4, D=5$ super-Yang-Mills theory with the multiplet (4) has a vector field $A_{M}$ and 5 scalars $\phi^{a b}=-\phi^{b a}, \Omega_{a b} \phi^{a b}=0(a, b=1, \ldots, 4)$, all in the adjoint of the gauge group. The central charges in the algebra (1) are the five electric 
charges

$$
Z^{a b}=\frac{1}{4 \pi} \int \operatorname{tr}\left(\phi^{a b} * F\right)
$$

integrated over a 3 -sphere at spatial infinity and the instanton charge

$$
K=\frac{4 \pi^{2}}{g_{Y M}^{2}} n_{I}, \quad n_{I}=\frac{1}{32 \pi^{2}} \int \operatorname{tr}(F \wedge F)
$$

integrated over a spatial hypersurface, where $n_{I}$ is the integral instanton number and $g_{Y M}$ is the Yang-Mills coupling constant. The W-bosons carry electric charge and fit into (1,1) massive vector multiplets while the 0-brane solitons in $4+1$ dimensions arising from lifting Yang-Mills instantons in 4 Euclidean dimensions carry the charge $K$ and fit into $(2,0)$ massive self-dual tensor multiplets. There is a tower of instantonic 0-branes with mass proportional to $\left|n_{I}\right| / g_{Y M}^{2}$ for all integers $n_{I}$ which become light in the strong coupling limit $g_{Y M} \rightarrow \infty$, signalling that an extra dimension is opening up, with the tower of massive states interpreted as a KaluzaKlein tower for a $(2,0)$ supersymmetric theory in 6 dimensisions compactified on a circle [4-6]. If a finite number of $(1,1)$ massive multiplets become massless at some point in moduli space, then there is an enhancement of the gauge symmetry at that point. If an infinite number become massless, this can signal a decompactification to a $(1,1)$ supersymmetric theory in 6 dimensions.

The little string theory in 6 dimensions with $(2,0)$ supersymmetry [5-10] compactified on a circle of radius $R$ has an infinite tower of momentum modes which are in $(2,0) \mathrm{D}=5$ massive multiplets and an infinite tower of winding modes, which are in $(1,1)$ multiplets. T-duality takes this to the $(1,1)$ little string theory on a circle with inverse radius $l_{s}^{2} / R$ for which the $(2,0)$ multiplets are now winding modes and the $(1,1)$ ones are momentum modes.

The $N=8$ supergravity theory in $D=5$ dimensions has the multiplet structure (6) including 27 abelian vector fields, and the electric charges for these (suitably dressed with scalars) give the 27 central charges $Z^{a b}$ in (1). The charge $K$ was defined in [2] and is carried by $1 / 2$-supersymmetric solutions of the form $N \times \mathbb{R}$ 
where $N$ is a self-dual gravitational instanton in 4 Euclidean dimensions [11] and $\mathbb{R}$ is time $[2,12]$. If $N$ is the multi-Taub NUT instanton, the solution has an interpretation as a multi-Kaluza-Klein monopole space [13], while if it is a multiEguchi Hansen space, the solution can be interpreted as representing a set of 0-branes in a 5-dimensional space-time [2].

It is straightforward to find the decomposition of each multiplet into representations of the little group $S U(2) \times S U(2) \times S p(p) \times S p(q)$, using e.g. the methods of [1]. For the three $1 / 2$-supersymmetric multiplets that could arise in $N=8$ supergravity (i.e. which reduce to $D=4$ multiplets with spins no greater than two), the results are as follows. The $(2,2)$ multiplet has the $S U(2) \times S U(2) \times S p(2) \times S p(2)$ content

$$
\begin{aligned}
& (3,3 ; 1,1)+(1,3 ; 5,1)+(3,1 ; 1,5)+(1,1 ; 5,5)+(2,2 ; 4,4) \\
& +(2,3 ; 4,1)+(3,2 ; 1,4)+(2,1 ; 4,5)+(1,2 ; 5,4)
\end{aligned}
$$

with

$$
\Lambda=\operatorname{diag}(\lambda, \lambda,-\lambda,-\lambda)
$$

so that

$$
K=0, \quad Z=\operatorname{diag}(\lambda, \lambda,-\lambda,-\lambda) \otimes\left(\begin{array}{cc}
0 & 1 \\
-1 & 0
\end{array}\right)
$$

The $(3,1)$ multiplet has the $S U(2) \times S U(2) \times S p(3) \times S p(1)$ content

$$
\begin{aligned}
& (4,2 ; 1,1)+(3,1 ; 6,2)+\left(1,1 ; 14^{\prime}, 2\right)+(2,2 ; 14,1) \\
& +(3,2 ; 6,1)+(2,1 ; 14,2)+\left(1,2 ; 14^{\prime}, 1\right)
\end{aligned}
$$

with

$$
\Lambda=\operatorname{diag}(\lambda,-\lambda,-\lambda,-\lambda)
$$

so that

$$
K=-4 \lambda, \quad Z=\operatorname{diag}(3 \lambda,-\lambda,-\lambda,-\lambda) \otimes\left(\begin{array}{cc}
0 & 1 \\
-1 & 0
\end{array}\right)
$$


The $(4,0)$ multiplet has the $S U(2) \times S U(2) \times S p(4)$ content

$$
(5,1 ; 1)+(3,1 ; 27)+(1,1 ; 42)+(4,1 ; 8)+(2,1 ; 48)
$$

with

$$
\Lambda=-\operatorname{diag}(\lambda, \lambda, \lambda, \lambda)
$$

so that $Z=0$ and $K=-8 \lambda$.

If $K=0$, the only possibilities for BPS states are those preserving $1 / 2$ the supersymmetry and fitting into massive $(2,2)$ multiplets, the $1 / 4$ supersymmetric states in $(3,3)$ supermultiplets and the $1 / 8$ supersymmetric states in $(4,3)$ or $(3,4)$ multiplets; all the other possibilities for $N=8$ that are listed in table 1 , including $3 / 8$ supersymmetry, cannot occur with $K=0$. The general BPS 0-brane or black hole solutions with $K=0$ were given in [14] by acting on the generating solution of [15] with U-duality transformations.

A non-zero value for $Z^{a b}$ necessarily breaks the $S p(4)$ to a subgroup. The instantonic solutions with $Z^{a b}=0$ and $K \neq 0$ fit into $(4,0)$ or $(0,4)$ multiplets, and the full $S p(4)$ is preserved. It is intriguing that the algebra also allows BPS states with both $K$ and $Z$ non-zero, and which would fit into multiplets with $(4,2),(4,1),(3,2)$ or $(3,1)$ supersymmetry. Of particular note is the unexpected $1 / 2$ supersymmetric $(3,1)$ multiplet, as $1 / 2$ supersymmetric states seem to play a special role in M-theory. The physics associated with these three $1 / 2$-supersymmetric multiplets will be discussed in a separate publication. 


\section{REFERENCES}

1. J. Strathdee, Int. Jour. Mod. Phys. A2 (1987) 273.

2. C.M. Hull, Nucl. Phys. B509 (1997) 252, hep-th/9705162.

3. M. Abou-Zeid, B. de Wit, D. Lust and H. Nicolai, Phys. Lett. B466 (1999) 144, hep-th/9908169.

4. M. Rozali, Phys.Lett. B400 (1997) 260, hep-th/9702136.

5. M. Berkooz, M. Rozali, and N. Seiberg, Phys.Lett. B408 (1997) 105, hepth/9704089.

6. N.Seiberg, hep-th/9705117.

7. E. Witten, in Proceedings of Strings 95, hep-th/9507121.

8. N. Seiberg, Phys.Lett. B408 (1997) 98, hep-th/9705221.

9. R. Dijkgraaf, E. Verlinde, and H. Verlinde, hep-th/9603126; hep-th/9604055.

10. O. Aharony, hep-th/9911147.

11. G.W. Gibbons and S.W. Hawking, Phys. Lett. 78B (1978) 430.

12. G.W. Gibbons and M.J. Perry, Nucl. Phys. B248 (1984) 629.

13. R. Sorkin, Phys. Rev. Lett. 51 (1983) 87 ; D. Gross and M. Perry, Nucl. Phys. B226 (1983) 29.

14. M. Cvetič and C.M. Hull, Nucl. Phys. B480 (1996) 296.

15. M. Cvetič and D. Youm, Nucl. Phys. B476 (1996) 118, hep-th/9603100. 